\title{
Development of Fingerprints for Quality Control of Acorus species by Gas Chromatography/Mass Spectrometry
}

\author{
Se Mi Yu, Eun Kyung Kim, Je Hyun Lee, ${ }^{\dagger}$ Kang Ro Lee, ${ }^{\star}$ and Jongki Hong \\ College of Pharmacy \& Kyung Hee East-west Pharmaceutical Research Institute, Kyung Hee University, Seoul 130-701, Korea \\ *E-mail: jhong@khu.ac.kr \\ ${ }^{\dagger}$ College of Korean Medicine, Dongguk University, Gyeongbuk 780-714, Korea \\ *College of Pharmacy, Sungkyunkwan University, Suwon 440-746, Korea \\ Received February 17, 2011, Accepted March 16, 2011
}

\begin{abstract}
An effective analytical method of gas chromatography/mass spectrometry (GC/MS) was developed for the rapid determination of essential oils in the crude extract of Acorus species (Acorus gramineus, Acorus tatarinowii, and Acorus calamus). Major phenypropanoids ( $\beta, \alpha$-asarone isomers, euasarone, and methyleugenol) and $\beta$-caryophyllene in Acorus species were used as marker compounds and determined for the quality control of herbal medicines. To extract marker compounds, various extraction techniques such as solvent immersion, mechanical shaking, and sonication were compared, and the greatest efficiency was observed with sonication extraction using petroleum ether. The dynamic range of the GC/MS method depended on the specific analyte; acceptable quantification was obtained between 10 and $2000 \mu \mathrm{g} / \mathrm{mL}$ for $\beta$-asarone, 10 and $500 \mu \mathrm{g} / \mathrm{mL}$ for $\alpha$-asarone, 10 and $200 \mu \mathrm{g} / \mathrm{mL}$ for methyleugenol, and between 5 and $100 \mu \mathrm{g} / \mathrm{mL}$ for $\beta$ caryophyllene. The method was deemed satisfactory by inter- and intra-day validation and exhibited both high accuracy and precision, with a relative standard deviation $<10 \%$. Overall limits of detection were approximately $0.34-0.83 \mu \mathrm{g} / \mathrm{mL}$, with a standard deviation $(\sigma)$-to-calibration slope $(\mathrm{s})$ ratio $(\sigma / \mathrm{s})$ of 3 . The limit of quantitation in our experiments was approximately $1.13-3.20 \mu \mathrm{g} / \mathrm{mL}$ at a $\sigma / \mathrm{s}$ of 10 . On the basement of method validation, 20 samples of Acorus species collected from markets in Korea were monitored for the quality control. In addition, principal component analysis (PCA) and hierarchical cluster analysis (HCA) were performed on the analytical data of 20 different Acorus species samples in order to classify samples that were collected from different regions.
\end{abstract}

Key Words : Acorus species, Essential oils, GC/MS, Principal component analysis (PCA), Hierarchical cluster analysis (HCA)

\section{Introduction}

Three kinds of Acorus species [Acorus gramineus (A.g.), Acorus tatarinowii (A.t.), and Acorus calamus (A.c.)] have been used as traditional Korean medicine, prepared from the dried rhizome (Acorus graminei rhizome, AGR) of the plant. Generally, the volatile constituents of Acorus species have been known to be the main source of the pharmacological activity of this medicine, including the as sedative, digestive, analgesic, diuretic, and antifungal agents properties. ${ }^{1,2}$ However, the chemical composition of herbal medicines is complex and active components are rarely identified. In addition, the quantities and contents of active compounds in herbal medicines are dependent on locations, intra-species variability, cultivating conditions, harvest period, storage time, and processing method., ${ }^{3,4}$ Besides these factors, the extraction methods used to process the herbal plants can also affect the quantities of biologically active compounds in the extract. It is not easy to determine the geographic origins with previously reported methods ${ }^{2,5}$ which were used to determine one or two components by chromatographic analysis. Thus, the development of methods for the quality control of multi-components in the herbal extract is of great importance in medicinal and dietary applications.

This herbal medicine contains several active components, including essential oils phenylpropanoids (e.g., $\beta$-asarone, $\alpha$-asarone, euasarone, methylisoeugenol, methyleugenol, and asarylaldehyde), terpenoids (e.g., caryophyllene, humulene, and safrole) as well as fatty acids and phenolic compounds. ${ }^{6,7}$ Among these components, the major components are known to be phenylpropanoids, including $\beta$-asarone, $\alpha$-asarone, euasarone, and methyleugenol. Sesquiterpenes including caryophyllene were also observed as characteristic components of Acorus species. These volatile components can be selected as marker compounds for quality control. The volatile components of Acorus species have been the subject of few investigations. ${ }^{5,8-10}$ For the analysis of volatile compounds in herbal medicines, gas chromatography (GC) and GC-mass spectrometry (GC/MS) have been widely used due to its high sensitivity and selectivelty. ${ }^{11,12}$ Moreover, the volatile compounds extracted from herbal medicines can be readily identified and quantified by GC/MS and the quantities of components can be used to assess certain characteristics of herbal medicines.

Several extraction methods, including steam distillation, ${ }^{13}$ solvent immersion extraction, ${ }^{14}$ and solid-phase extraction, ${ }^{15}$ 
have been used for the quantitative extraction of volatile compounds from a sample matrix. However, these methods are laborious, time consuming, and could result in the loss of important volatiles during sample workup. Recently, solidphase microextraction (SPME) and purge-and-trap methods using various adsorbents have been widely used for the rapid extraction of volatile compounds from aromatic and medicinal plants. ${ }^{16-18}$ SPME has been proven as a useful extraction method, with the advantages of rapidity and simplicity as well as no use of organic solvents. However, several parameters in SPME, such as the times and temperatures of adsorption and desorption process, kind of fibers, and sample amounts, should be optimized prior to sample analysis. Moreover, accurate quantification of biologically active compounds cannot be successfully obtained with this method, owing to the lack of a proper internal standard. For the rapid extraction of biologically active or marker compounds from herbal medicine, sonication extraction has been also introduced $^{19,20}$ and is useful due to its easy operation as well as its use of less organic solvent. Moreover, this method has successfully been used for the extraction of organic compounds in biological tissue samples and environmental matrices. ${ }^{21-23}$

To evaluate the quality of Acorus species, the quantification of one, or even several, of the compounds in an herbal medicine were currently employed. However, this kind of the determination is not considered an adequate approach for quality control. The quality of Acorus species can be accurately predicted by the analysis of several marker compounds, even though not be related with the pharmacological effect of herbal medicine. Therefore, chemical fingerprint analysis can evaluate the quality control of herbal medicines and the classification of geographic origins. Pattern recognition analysis using multivariate statistical analysis has been extensively used, and this approach provides information on the overall chemical composition of herbal medicines. In addition, principal component analysis (PCA) can be used to provide a convenient visual aid for the identification of inhomogeneity in the data sets. ${ }^{19,20,24-28}$ Due to its usefulness in the differentiation of samples, PCA has been recently applied to the classification of traditional herbal plants from different origins. ${ }^{26-29}$ The PCA technique, combined with chromatographic data of selected marker compounds, can be successfully applied in the quality control of herbal extracts. ${ }^{19,20,29,30}$ Moreover, hierarchical cluster analysis (HCA) has been used to sort samples into groups by measuring similarity between samples. The similarity and dissimilarity between samples can be represented in dendrogram for ease interpretation.

The objective of this work was to demonstrate that the GC/MS method, combined with sonication extraction, can be used as a rapid and effective method for the characterization of Acorus species samples. A total of 20 Acorus species samples were collected from the market and analyzed using the validated analytical method. These chromatographic data were then applied in the statistical analysis of herbal medicine for quality control. The classification of these 20
Acorus species samples collected from different regions was performed using PCA and HCA combined with chromatographic fingerprints.

\section{Experiments}

Reagents and Apparatus. All reagents that were used in this study were of analytical grade. Petroleum ether and methanol were purchased from J.T. Baker (Phillipsburg, NJ, USA). Some of volatile components, such as $\alpha$-asarone, and methyleugenol were purchased from Aldrich (Milwaukee, WI, USA, purity $\geq 98 \%$ ). $\beta$-Asarone and $\beta$-caryophyllene were purchased from Carl Roth (purity $\geq 98 \%$, Karlsruhe, Germany) and Wako Chemical (purity $\geq 90 \%$, Osaka, Japan), respectively.

The chemical structures of these major compounds are shown in Figure 1. Analytical samples of the herbal medicine, Acorus species were purchased from various market places in Korea and China and were identified by an expert discriminator of herbal medicine. Prior to use, the Acorus species was dried and pulverized, then filtered using a standard sieve with a mesh size of 30 .

Sample Preparation. Dried rhizome powders were used for each extraction to determine the content of the marker compounds and to perform chemical fingerprint analysis of Acorus species samples. Dried rhizome of Acorus species was pulverized and $1.0 \mathrm{~g}$ was placed into $20 \mathrm{~mL}$ of petroleum ether in a volumetric flask. For solvent immersion extraction, the sample mixture was allowed to stand for $24 \mathrm{~h}$ at room temperature in a volumetric flask. For sonication extraction, the sample mixture was extracted for $10 \mathrm{~min}$ period in an ultrasonic bath at room temperature. After extraction, $3 \mathrm{~g}$ of sodium sulfate were added to the sample mixture to remove trace water. The sample mixture was then filtered through a $0.2 \mu \mathrm{m}$ membrane filter and carefully dried under a stream of nitrogen to a final volume of $5 \mathrm{~mL}$. $50 \mu \mathrm{L}$ of internal standard was then added to this solution and a $1-\mu \mathrm{L}$ sample of the solution was injected into the GC/ MS system. The overall analytical procedure for the determination of the marker compounds in Acorus species is

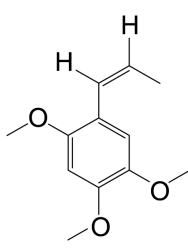

$\alpha$-asarone

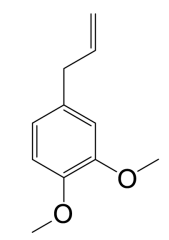

methyleugenol

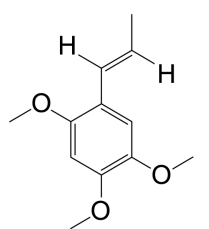

$\beta$-asarone

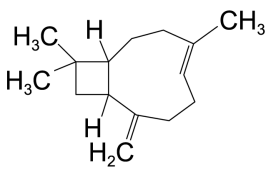

$\beta$-caryophyllene 1,4-dimethoxybenzene (IS)
Figure 1. Chemical structures of the marker compounds and the internal standard (IS) used in this study. 


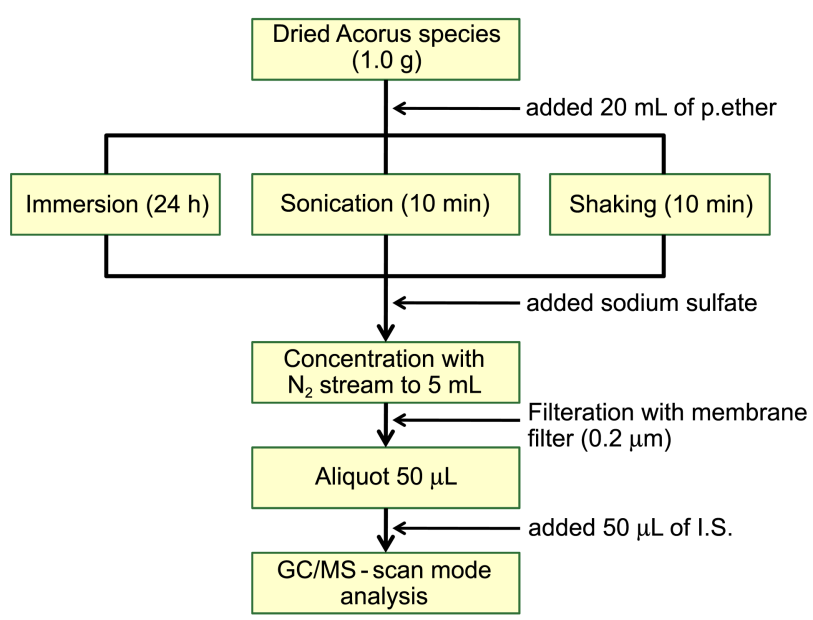

Figure 2. Overall analytical procedure for extraction of marker compounds from Acorus species.

\section{shown in Figure 2.}

Gas Chromatography-mass Spectrometry. GC/MS analyses were carried out with an Agilent $6890 \mathrm{~N}$ gas chromatography instrument, combined with an Agilent-5973 mass selective detector that was equipped with an electron ionization and quadrupole analyzer. The electron ionization energy was set at $70 \mathrm{eV}$. The temperatures of the ion source and interface were set at 230 and $280^{\circ} \mathrm{C}$, respectively. The mass scan range was from 40 to $400 \mathrm{amu}$. The separation of volatile compounds was carried out with a $5 \%$ phenyl dimethylpolysiloxane fused-silica capillary column (DB-5MS $30 \mathrm{~m} \times 0.25 \mathrm{~mm}$ i.d., film thickness $0.25 \mu \mathrm{m}$, J\&W Scientific, Folsom, CA, USA). A split injection (split ratio, 20:1) and injector temperature of $270{ }^{\circ} \mathrm{C}$ were employed. The oven temperature was programmed to increase from 80 (for $3 \mathrm{~min}$ ) to $90^{\circ} \mathrm{C}$ at a rate of $10{ }^{\circ} \mathrm{C} / \mathrm{min}$, then increase from 90 to $140^{\circ} \mathrm{C}$ at a rate $10^{\circ} \mathrm{C} / \mathrm{min}$, and then increase from 140 to $280^{\circ} \mathrm{C}$ at a rate $20^{\circ} \mathrm{C} / \mathrm{min}$.

Principal Component Analysis (PCA) and Hierarchical Clustering Analysis (HCA). PCA and HCA were performed using the singular value decomposition method by the Multivariate Statistical Package program (MVSP, Kovach Computing Service, Anglesey, Wales). For the classification of herbal medicines, PCA and HCA were performed by applying the detected peak area of marker compounds among volatile compounds that were obtained from the GC/MS analysis of 20 Acorus species samples. For pattern recognition analysis, 6 major peaks that were observed in the total ion chromatogram were selected and their peak areas were calculated.

\section{Results and Discussion}

Extraction of Marker Compounds from Acorus species. In the extraction of marker compound from Acorus species, both the selection of solvent and the extraction method can be critical in obtaining a satisfactory extraction of marker compounds. Also, these factors can be important in obtaining a good chromatographic fingerprint representing the

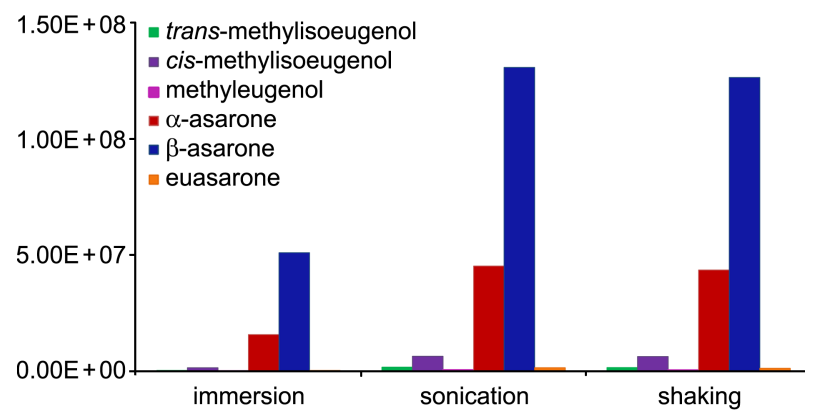

Figgure 3. Comparison of contents of marker compounds in Acorus species obtained from three different extraction methods.

quality control of herbal medicine. In other words, the chemical compositions of extract can be significantly changed by both factors. In this study, $n$-hexane, methanol, ethanol, and petroleum ether were used as extraction solvents. No significant difference was found among solvents used in extraction, probably due to their good dissolving capability for volatiles. Methanol and ethanol have relatively high boiling points and thus were not suitable in this method as some of volatiles could be lost during evaporation. Both $n$ hexane and petroleum ether are suitable for the extraction of volatiles, but $n$-hexane exhibits relatively lower extraction efficiency for some compounds as compared to petroleum ether. Moreover, petroleum ether could be easily applied for sample workup due to its low boiling point.

Three extraction methods including solvent immersion extraction, sonication extraction, and shaking methods were tested and compared, as depicted in Experiments section. The extraction amounts of marker compounds from Acorus species, categorized by extraction method, are shown in Figure 3. Shaking extraction and solvent immersion are the conventional method for the extraction of herbal medicines, but these methods require a longer extraction time and can be labor-intensive. The sonication method had advantages for rapid extraction using less organic solvent. As can be seen in Figure 3, the solvent immersion method was shown to be a relatively poor method for the recovery of marker compounds. The sonication was shown to be most effective at the extraction of phenylpropanoids. However, this method could lead to a loss of volatiles if sonication period was longer than 30 successive minutes, due to an increase in the temperature. In this study, sonication extraction using $20 \mathrm{~mL}$ of petroleum ether was performed for $10 \mathrm{~min}$, to protect against the loss of volatiles.

Chemical Fingerprints of Acorus species. Typical total ion chromatograms of the extracts of Acorus species including rhizomes of A.g., A.t., and A.c. and radix of A.g. obtained by GC/MS-scan mode are shown in Figure 4. Fingerprints for rhizomes of A.g. and A.t. showed apparently similar chromatographic patterns, but exhibited significant different form fingerprints for rhizomes of A.c. and radixs of A.g. Actually, these species can be easily discriminated by difference in their morphology. However, rhizomes of A.g. and A.t. which are widely used for medicinal purpose in Korea could not be easily differentiated due to their morphological 

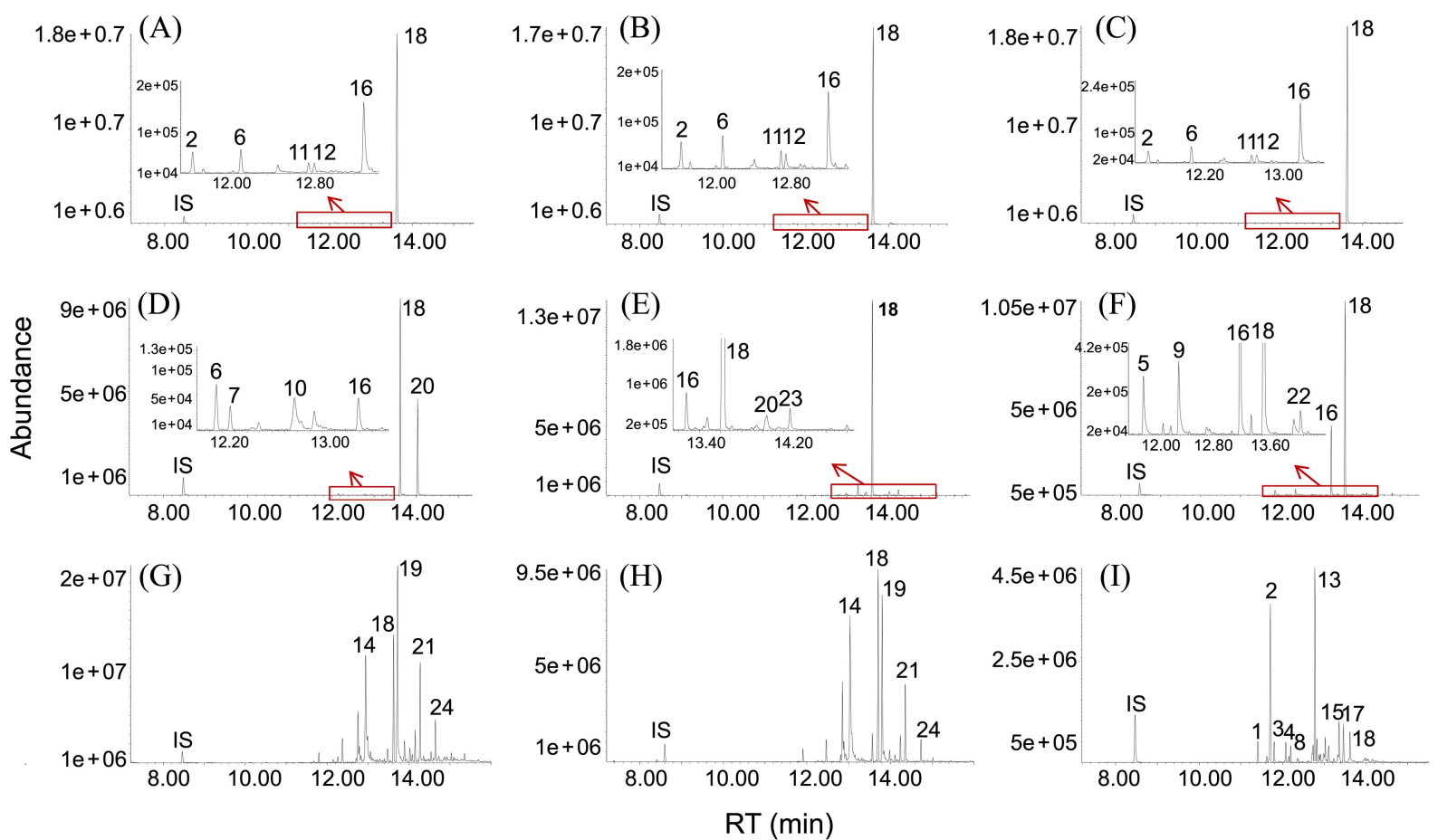

Figure 4. Typical total ion chromatograms of the extracts of different Acorus species samples; rhizome of Acorus gramineus solander (A-C), rhizome of Acorus tatarinowii schott (D-F), rhizome of Acorus calamus L. (G and H), and radix of Acorus gramineus solander (I). Peak identities are as follows; 1 : $\alpha$-longipinene, 2 : longicyclene, 3: $\beta$-elemene, 4: junipene, 5 : methyleugenol, 6 : $\beta$-caryophyllene, 7: calarene, 8 : $\gamma$-elemene, 9: cis-methyleugenol, 10: trans-methylisoeugenol, 11: $\gamma$-gurjunene, 12: $\alpha$-muurolene, 13: $\alpha$-gurjunene, 14: cyclohexanone, 3 ethenyl-3-methyl-2-(1-methylethenyl)-6-(1-methylethyl-), 15: $\beta$-guaiene, 16: euasarone, 17: eudesmol, 18: $\beta$-asarone, 19: 8-(1-pentenyl)carbonyl)bicyclo[6.1.0]nonane, 20: $\alpha$-asarone, 21: unknown, 22: (+)-3,8-dimethyl-5(1-methylethylidene)-octahydroazulene-6-one, 23: 1,4cis-1,7-trans acorenone, and 24: isocalamendiol.

similarity. Therefore, the chemical profiles of volatiles observed in Acorus species could be characterized by the amounts of the predominant components. To evaluate the quality control of herbal medicines, the concentration level of main and/or characteristic components should be preferentially identified and measured.

As can be seen in Figure 4, total 24 volatile components in Acorus species were identified by GC/MS-scan mode. Among them, phenylpropanoid compounds including $\alpha$-, $\beta$-asarone isomers, euasarone, and methyleugenol isomers, were observed as the major compounds. These compounds were identified by the comparison of retention times and mass spectra of authentic standards. The contents of these phenylpropanids comprised above $90 \%$ of the total amount of volatile compounds in A.g. and A.t. In this study, the chemical compositions of volatiles observed in rhizomes of A.g. and A.t. were mainly consisted of phenylpropanoids (about 99 and 94\%), sesquiterpenes (about 1 and 3\%), and others (about
0.0 and $3 \%$ ), as indicated in Table 2 . On the other hand, the chemical compositions of rhizome of A.c. are consisted of sesquiterpenes $(6 \%)$, monoterpenes and others $(74 \%)$, and phenylpropanoids (20\%). The chemical composition of radix of A.g. showed unique distributions, taking $27 \%$ of phenylpropanoids and $73 \%$ of sesquiterpenes. Especially, longicyclene (peak \#2) and $\alpha$-gurjunene (peak \#13) of sesquiterpenes were observed as major compounds in radix of A.g.

Method Validation. To evaluate the quality control of Acorus species, the method validation should be performed to determine volatile marker compounds in herbal plant. In this study, linearity, quantification limit, accuracy and precision, and application to herbal medicine were tested for analytical method validation.

Calibration curves were generated using standard solutions, containing 10 and $2000 \mu \mathrm{g} / \mathrm{mL}$ for $\beta$-asarone, 10 and $500 \mu \mathrm{g} / \mathrm{mL}$ for $\alpha$-asarone, 10 and $200 \mu \mathrm{g} / \mathrm{mL}$ for methyleugenol, and between 5 and $100 \mu \mathrm{g} / \mathrm{mL}$ for $\beta$-caryophyllene.

Table 1. Equation of calibration curves, linearity correlation coefficients, LODs, and LOQs for marker compounds

\begin{tabular}{|c|c|c|c|c|c|c|}
\hline Compounds & $\begin{array}{l}\text { Linear range } \\
\quad(\mu \mathrm{g} / \mathrm{g})\end{array}$ & Slope & Intercept & $\begin{array}{r}\text { Correlation } \\
\text { coefficient }\end{array}$ & LOD & LOQ \\
\hline$\alpha$-asarone & $10.0-500.0$ & 0.0226 & -0.3958 & 0.996 & 0.80 & 3.20 \\
\hline methyleugenol & $10.0-200.0$ & 0.0147 & -0.1724 & 0.997 & 0.77 & 2.91 \\
\hline$\beta$-caryophyllene & $5.0-100.0$ & 0.0152 & -0.0506 & 0.998 & 0.34 & 1.13 \\
\hline
\end{tabular}


Table 2. Amounts of volatile types in Acorus species detected by GC/MS-scan mode

\begin{tabular}{|c|c|c|c|c|c|}
\hline & & \multicolumn{2}{|c|}{ A.g. solan. (\%) } & \multirow{2}{*}{$\frac{\text { A.t.schott }(\%)}{\text { rhizome }}$} & \multirow{2}{*}{$\frac{\text { A.c.lin. }(\%)}{\text { rhizome }}$} \\
\hline \multirow{7}{*}{ Phenylpropanoids } & compounds & rhizome & radix & & \\
\hline & $\alpha$-asarone & 1.05 & 1.12 & 4.23 & - \\
\hline & $\beta$-asarone & 97.07 & 25.85 & 79.91 & 17.67 \\
\hline & euasarone & 0.84 & - & 7.30 & - \\
\hline & trans-methylisoeugenol & - & - & 0.02 & - \\
\hline & cis-methylisoeugenol & 0.19 & - & 2.78 & 2.64 \\
\hline & methyleugenol & - & - & 0.55 & - \\
\hline \multirow{8}{*}{ Sesquiterpenes } & longicyclene & 0.38 & 12.05 & 0.05 & - \\
\hline & $\beta$-elemene & - & 4.45 & 0.09 & 1.27 \\
\hline & $\beta$-caryophyllene & 0.22 & 0.30 & 0.17 & - \\
\hline & calarene & - & 17.41 & 0.15 & 0.34 \\
\hline & $\gamma$-gurjunene & 0.15 & 16.59 & - & - \\
\hline & 1,4-cis-1,7-trans-acorenone & - & 4.54 & 0.81 & - \\
\hline & isocalmendiol & - & - & 0.86 & 3.50 \\
\hline & others & 0.09 & 17.69 & 0.06 & 0.51 \\
\hline Others & unknown and etc. & - & - & 3.01 & 74.11 \\
\hline
\end{tabular}

These calibration ranges were found to be adequate for the quantification of marker compounds in herbal extracts. Four standard solutions were analyzed in triplicate, and multipoint calibration curves were constructed by linear regression analysis of the peak area ratios of each analyte, as compared to the internal standard, versus concentration. The calibration curves of the analytes showed good linearity within given concentration ranges. Correlation coefficients ranged from 0.996 to 0.998 . Equations representing the calibration curves and their correlation coefficients are summarized in Table 1.

The limit of detection (LOD) and limit of quantification (LOQ) were calculated by statistical methods ${ }^{31}$ using the ratio of three and ten, respectively, standard deviations $(\sigma)$ of response and slope (s) of the calibration curve. LODs and LOQs of four marker compounds were determined by performing 10 injections of each compound at concentrations incrementally approaching the LOD and LOQ. These LOD and LOQ values ranged from 0.34 to 0.83 and from 1.13 to $3.20 \mu \mathrm{g} / \mathrm{mL}$, respectively, for the four marker compounds, demonstrating a reasonable sensitivity. In many cases of GC/ MS analysis, the LOD and LOQ could be greatly decreased by adjusting the sample volume, the detection mode, such as scan or selected ion monitoring, and the injection mode. The LODs and LOQs of four marker compounds are also represented in Table 1. These compounds were detected with reasonable sensitivity for the quality control of Acorus species. In other words, these LOQ ranges were shown to be detectable at approximately 2-10 fold lower concentrations than those present in the herbal medicine.

To test the accuracy and precision of the analytical method, four marker compounds were added, at concentrations of 25 , 50, and $75 \mu \mathrm{g} / \mathrm{mL}$, into Acorus species samples. The precision and accuracy within the day were determined from the variability of replicate analyses of spiked herbal medicine within the same analytical run. Inter-day precision and accuracy were evaluated from the variability of replicate analyses of spiked herbal medicine for five consecutive days. The precision of the method for simultaneously determining the four marker compounds was acceptable, as the relative standard deviation (RSD) did not exceed 10\% at concentrations of 25,50 , or $75 \mu \mathrm{g} / \mathrm{mL}$. At the same concentrations, the intra-day accuracy ranged from 95.9 to $102.1 \%$, whereas the inter-day accuracy ranged from 92.4 to $104.6 \%$, indicating an excellent accuracy. These inter-day and intra-day data demonstrate that the developed method is highly reproducibility and precise for our four marker compounds.

Application of the Method. The method developed herein was subsequently applied to 20 different samples of Acorus species for the simultaneous determination of the five marker compounds. Here, it is assumption that the calibration equation of euasarone is as same as that of $\beta$-asarone because euasarone is not commercially available and major component. The amounts of marker compounds in Acorus species were determined in samples collected from 8 regions of China (C-1 to C-8) and from 12 regions of Korea (K-1 to K12). Each sample was analyzed in five repetitions to ensure the reproducibility of the quantitative result.

As shown in Figure 4, total ion chromatograms of the extracts of Acorus species were indicated the absence of any significant interference in the quantitation of the five marker compounds. A summary of the regional data is shown in Table 3, and these results show significant geographical origin variability. The data indicated that marker compounds were detected in almost analyzed samples. For most of Acorus species samples, $\beta$-asarone was found to be the most abundant component. $\alpha$-Asarone, euasarone, and $\beta$-caryophyllene were detected in almost samples. Methyleugenol was detected in A.t. samples except for C-6 but not observed in all A.g. soland samples. Therefore, the amounts of $\beta, \alpha-$ asarones isomers, euasarone, $\beta$-caryophyllene, and methyleugenol, could be used as the most important marker com- 
Table 3. Amounts of the five marker compounds in Acorus species samples cultivated from different regions $(\mathrm{n}=3)$

\begin{tabular}{|c|c|c|c|c|c|}
\hline \multirow{2}{*}{$\begin{array}{l}\text { Sample } \\
\text { number }\end{array}$} & \multicolumn{5}{|c|}{ Mean concentration $(\mathrm{ppm}) \pm \mathrm{RSD}$} \\
\hline & $\alpha$-asarone & $\beta$-asarone & euasarone & methyleugenol & $\beta$-caryophyllene \\
\hline${ }^{a} \mathrm{~K}-1$ & $25.44 \pm 0.92$ & $1064.31 \pm 1.54$ & $4.33 \pm 6.66$ & - & $6.22 \pm 3.72$ \\
\hline $\mathrm{K}-2$ & $25.71 \pm 0.90$ & $772.69 \pm 0.95$ & $0.96 \pm 9.53$ & - & $6.28 \pm 0.72$ \\
\hline $\mathrm{K}-3$ & $25.48 \pm 1.27$ & $793.78 \pm 1.46$ & $1.91 \pm 9.94$ & - & $6.48 \pm 0.68$ \\
\hline $\mathrm{K}-4$ & $25.58 \pm 0.49$ & $922.84 \pm 0.10$ & $3.28 \pm 6.74$ & - & $5.66 \pm 1.79$ \\
\hline $\mathrm{K}-5$ & $22.85 \pm 0.47$ & $727.53 \pm 0.59$ & $0.46 \pm 6.33$ & - & $4.38 \pm 3.00$ \\
\hline $\mathrm{K}-6$ & - & $1.67 \pm 16.13$ & - & - & - \\
\hline $\mathrm{K}-7$ & $21.50 \pm 0.73$ & $640.57 \pm 0.52$ & $0.53 \pm 6.14$ & - & $6.87 \pm 0.09$ \\
\hline $\mathrm{K}-8$ & - & $23.30 \pm 4.75$ & - & - & $9.06 \pm 0.12$ \\
\hline K-9 & - & $400.13 \pm 0.28$ & - & - & $16.40 \pm 0.38$ \\
\hline $\mathrm{K}-10$ & - & $405.34 \pm 0.95$ & - & - & $7.72 \pm 2.07$ \\
\hline K-11 & $23.77 \pm 0.63$ & $602.63 \pm 0.81$ & - & - & - \\
\hline $\mathrm{K}-12$ & $19.81 \pm 0.15$ & $28.46 \pm 0.13$ & - & - & - \\
\hline${ }^{b} \mathrm{C}-1$ & $23.88 \pm 1.39$ & $549.88 \pm 1.87$ & $181.69 \pm 1.14$ & $40.82 \pm 3.59$ & $6.84 \pm 10.93$ \\
\hline $\mathrm{C}-2$ & $37.23 \pm 3.77$ & $529.00 \pm 1.06$ & $19.19 \pm 2.72$ & $15.55 \pm 0.37$ & $7.07 \pm 0.97$ \\
\hline $\mathrm{C}-3$ & $46.44 \pm 0.72$ & $988.49 \pm 0.60$ & $14.33 \pm 2.88$ & $13.78 \pm 0.34$ & $9.47 \pm 0.92$ \\
\hline C-4 & $39.67 \pm 1.49$ & $962.32 \pm 1.24$ & $10.95 \pm 6.27$ & $12.94 \pm 0.98$ & - \\
\hline C-5 & $35.04 \pm 0.42$ & $605.63 \pm 0.31$ & $24.84 \pm 0.98$ & $15.93 \pm 0.10$ & - \\
\hline C-6 & $53.55 \pm 0.89$ & $788.31 \pm 0.25$ & $8.13 \pm 1.73$ & - & $8.64 \pm 0.20$ \\
\hline $\mathrm{C}-7$ & $33.76 \pm 0.16$ & $588.04 \pm 0.15$ & $187.02 \pm 0.36$ & $15.42 \pm 0.42$ & $5.80 \pm 0.22$ \\
\hline $\mathrm{C}-8$ & $26.05 \pm 2.40$ & $338.94 \pm 2.99$ & $267.68 \pm 2.54$ & $67.41 \pm 4.41$ & - \\
\hline
\end{tabular}

${ }^{a} \mathrm{~K}-1 \sim 12$ : Korean sample; rhizome of $A . g . ;$ K-1,5,11: Jeju, K-2: Jangsu, K-3: Namduk usan, K-4: Gurye, and K-7: Seoul; radix of $A . g . ;$ K-6,12: Jeju and K-8: Seoul (radix); rhizome of A.c.lin.; K-9: Gyeongju and K-10: Seoul. ${ }^{b} \mathrm{C}-1 \sim 8$ : Chinese sample; rhizome of A.t.; C-1: Anhui, C-8: Chengdu, C-3,5; Sichuan, C-4: Hubei, C-6: Jiangxi, and C-2,7: not specified a collected region

pounds in pattern recognition analysis.

Quality Assessment by PCA and HCA. To classify the herbal plants with different geographical origins, 20 Acorus species samples were analyzed by established method, to obtain chromatographic fingerprints. These fingerprints showed similar chromatographic patterns and quiet different patterns according to geographic origins and medicinal parts. These differences may be a result of various factors includ-

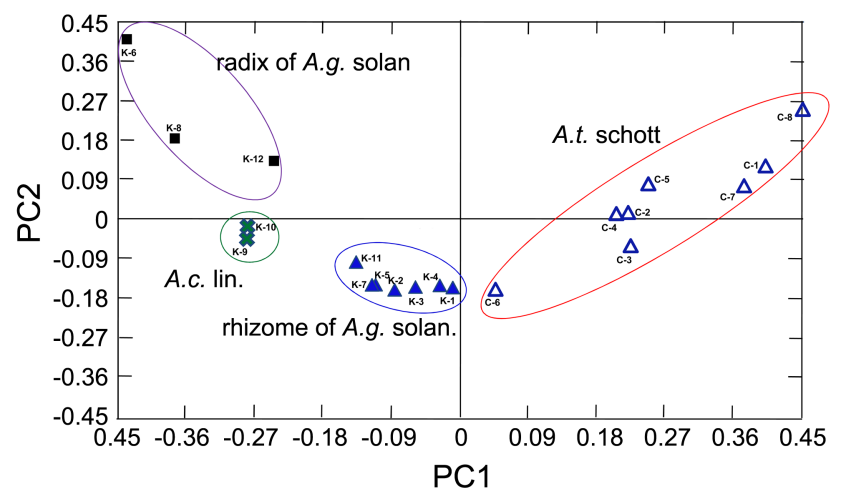

Figure 5. Represetation of 20 chromatographic samples of Acorus species on PC1 and PC2 (83.92\% variance explained). K-1 12: Korean sample; K-1,5,11: Jeju (rhizome), K-6,12: Jeju (radix), K2: Jangsu (rhizome), K-3: Namduk (rhizome), K-4: Gurye (rhizome), K-9: Gyeongju (rhizome of A.c.lin.), K-7: Seoul (rhizome), K-10: Seoul (rhizome of A.c.lin.), and K-8: Seoul (radix). C-1 8: Chinese sample; C-1: Anhui (rhizome), C-8: Chengdu (rhizome), C-3,5; Sichuan (rhizome), C-4: Hubei (rhizome), C-6: Jiangxi (rhizome), and C-2,7: not specified a collected region (rhizome). ing origins, cultivation region and soil, medicinal parts, and storage conditions. PCA using the peak area of the five marker compounds was performed on the analytical data of all 20 samples. To display the points in two principal components, PC 1 and PC 2 (first and second principal components) were chosen to represent the information. Figure 5 shows the principal component projection plot of the PC 1 and PC 2 of 20 Acorus species samples. It was found that PC 1 and PC 2 reflected $83.92 \%$ of the total variance in these samples. From the scatter points, the samples could be classified into four groups, indicating a clear differentiation between Acorus species collected in different geographic origins, except for C-6. Fingerprint of C-6 was almost similar to those of rhizome of A.g. samples. The scattered points of A.g. solan samples were closely gathered at center of PCA plot due to their chromatographic similarity. On the other hand, the scattered points of A.t. samples were widely distributed on right side due to their different chromatographic patterns. The scatter points of radix of $A . g$. samples were distributed at upper of right side and those of A.c. samples showed middle of left side.

To differentiate the Acorus species samples collected from market, HCA was also applied to the chromatographic data that was obtained from all samples. Peak area ratios of the marker compounds were calculated by the peak area ratio of analytes and an internal standard. Figure 6 shows the dendrogram of HCA, demonstrating that the similarity characteristics of 20 Acorus species could be revealed more clearly. The similarity between 20 samples was closely related with 


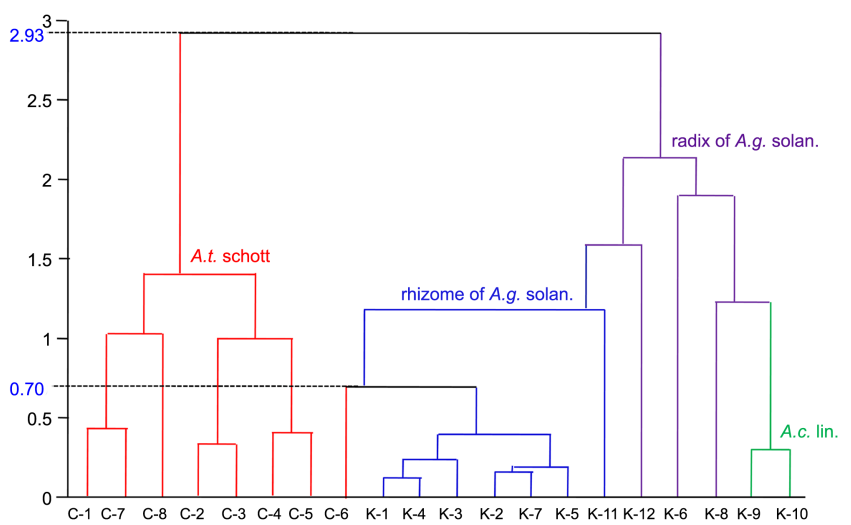

Figure 6. Dendrogram of Acorus species generated by weighted pair group method based on hierarchical cluster analysis.

the amounts of five marker compounds in Acorus species. Similar to the PCA results, samples could be mainly classified into four groups. Distance with 2.2 demonstrates the differentiation of medicinal part for radix and rhizome. Korea and China samples could be further classified into three subcluster set with high similarity of 0.7 and 1.2 distance, which demonstrates the differentiation of geographic origins (A.g., A.t., and A.c.) providing the information of geographical origins of herbs. However, C-6 sample (rhizome of A.t.) was belonged in rhzome of A.g. group due to not observing methyleugenol. At the present time, the unique specificity of C-6 could not be clearly explained and need further study for classification.

Therefore, this result demonstrated that PCA and HCA can provide visual differentiation according to cultivation regions and use complement data for the conventional quality control of herbal medicines.

\section{Conclusion}

In this study, we described a simple and rapid GC/MS method for quantifying major volatile compounds in Acorus species. The sonication method was shown to be rapid and effective for the extraction of volatiles from herbal medicine. Our validation data indicates that the developed analytical method is suitable for the quantification of marker compounds and the chemical standardization of herbal medicines obtained from Acorus species, regardless of the geographic origin. The simplicity of the procedure, combined with its reasonable sensitivity, resolution, and short analysis time, makes this method a useful tool for characterizing volatile compounds in other herbal medicines. In addition, PCA and HCA can provide important information on the differentiation of herbal plants from different regions. This developed GC/MS method, combined with multivariate statistical analysis, is a promising prospective methodology for obtaining comprehensive quality control of related herbal medicines and aromatic plants.

Acknowledgments. This study was supported by grants from the Korea Food and Drug Administration (2008) for
"Studies on the standardization of herbal medicines" and the Basic Research program (2009-0090546) of the National Research Foundation of Korea.

\section{References}

1. Tang, W.; Eisenbrand, G. Chinese Drugs of Plant Origin; Springer: New York, 1992; 45.

2. Chinese Pharmacopoeia Committee, Chinese Pharmacopoeia 1; Chemical Industry Press: Beijing, China, 2010; p 85.

3. Dubey, N. K.; Kumar, R.; Tripathi, P Curr. Sci. 2004, 86, 37.

4. Hendriks, M. M. W. B.; Juarez, L. C.; Bont, D. D.; Hall, R. D. Anal. Chim. Acta 2005, 545, 53.

5. Oprean, R.; Tamas, M.; Roman, L. J. Pharm. Biomed. Anal. 1998, 18, 227.

6. Oprean, R.; Oprean, L.; Tamas, M.; Sandulescu, R.; Roman, L. J. Pharm. Biomed. Anal. 2001, 24, 1163.

7. Wang, H. Z.; Chen, Y.; Fan, C. Acta Bot. Yunnanica Suppl. 1998, $5,96$.

8. Ye, H.; Ji, J.; Deng, C.; Yao, N.; Li, N.; Zhang, X. Chromatographia 2006, 63, 591 .

9. Deng, C.; Li, N.; Zhang, X. J. Chromatogr. A 2004, 1059, 149.

10. Dai, J.; Ha, C.; Shen, M. J. Sep. Sci. 2008, 31, 714.

11. Agnihotri, V. K.; Agarwal, S. G.; Dhar, P. L.; Thappa Baleshwar, R. K.; Kapahi, B. K.; Saxena, R. K.; Qazi, G. N. Flavour Fragance J. 2005, 20, 607.

12. Zhang, Y.; Wang, Z. Z. J. Pharm. Biomed. Anal. 2008, 47, 213.

13. Benzo, M.; Gilardoni, G.; Gandini, C.; Caccialanza, G.; Finzi, P. V.; Vidari, G.; Abdo, S.; Layedra, P. J. Chromatogr. A 2007, 1150, 131.

14. Gherman, C.; Culea, M.; Cozar, O. Talanta 2000, 53, 253.

15. Bertoli, A.; Pistelli, L.; Morelli, I.; Fraternale, D.; Giamperi, L.; Ricci, D. Flavour Fragance J. 2004, 19, 522.

16. Cuia, S.; Tan, S.; Ouyang, G.; Jiang, S.; Pawliszyn, J. J. Chromatogr. B 2009, 877, 1901 .

17. Stashenko, E. E.; Martínez, J. R. J. Biochem. Biophys. Methods 2007, 70, 235 .

18. Oh, S. Y.; Shin, H. D.; Kim, S. J.; Hong, J. J. Chromatogr. A 2008 , 1183,170

19. Lee, M. K.; Ahn, Y. M.; Lee, K. R.; Jung, J. H.; Jung, O. S.; Hong, J. Anal. Chim. Acta 2009, 633, 271.

20. Lee, M. K.; Ling, J. H.; Chun, M. H.; Jeong, J. H.; Na, Y. C.; Lee, K. W.; Jung, J. H.; Hong, J. Bull. Korean Chem. Soc. 2008, 29, 2465.

21. Seo, J.; H. Kim, Y.; Chung, B. C.; Hong, J. J. Chromatogr. A 2005, 1067, 303.

22. Hong, J.; Kim, H. Y.; Kim, D. G.; Seo, J.; Kim, K. J J. Chromatogr. A 2004, 1038, 27.

23. Kim, M. S.; Kang, T. W.; Pyo, H.; Yoon, J.; Choi, K.; Hong, J. J. Chromatogr. A 2008, 1208, 25.

24. Hadian, J.; Ebrahimi, S. N.; Salehi, P. Ind. Crop. Prod. 2010, 32, 62.

25. Liang, X.; Jin, Y.; Wang, Y.; Jin, G.; Fu, Q.; Xiao, Y. J. Chromatogr. A 2009, 1216, 2033

26. Telci, I.; Bayram, E.; Yilmaz, G.; Avci, B. Biochem. Sys. Ecol. 2006, 34, 489

27. Oh, S. Y.; Ko, J. W.; Jeong, S. Y.; Hong, J. J. Chromatogr. A 2008 , $1205,117$.

28. Zeng, Z. D.; Liang, Y. Z.; Chau, F. T.; Chen, S.; Daniel, M. K. W.; Chan, C. O. Anal. Chim. Acta 2007, 604, 89.

29. Yan, S.; Luo, G.; Wang, Y.; Cheng, Y. J. Pharm. Biomed. Anal. 2006, 40, 889 .

30. Li, B. Y.; Hu, Y.; Liang, Y. Z.; Xie, P. S.; Du, Y. P. Anal. Chim. Acta 2004, 514, 69.

31. ICH, Q2B, Validation of analytical procedure: methodology, in: Proceedings of the International Conference on Harmonization, London, 1996. 